\title{
Beryllium Bonding in the Light of Modern Quantum Chemical Topology Tools
}

José Luis Casals-Sainz, Fernando Jiménez-Grávalos, Aurora Costales, Evelio Francisco, and Ángel Martín Pendás*

Departamento de Química Física y Analítica. Universidad de Oviedo. Spain.

E-mail: ampendas@uniovi.es

${ }^{*}$ To whom correspondence should be addressed 


\begin{abstract}
We apply several modern quantum chemical topology (QCT) tools to explore the chemical bonding in well established Beryllium bonds. By using the interacting quantum atoms (IQA) approach together with electron distribution functions (EDF) and the natural adaptive orbitals (NAdOs) picture we show that, in agreement with orbital-based analyses, the interaction in simple $\sigma$ and $\pi$ complexes formed by $\mathrm{BeX}_{2}(\mathrm{X}=\mathrm{H}, \mathrm{F}, \mathrm{Cl})$ with water, ammonia, ethylene and acetylene is dominated by electrostatic terms, albeit covalent contributions cannot be ignored. Our detailed analysis proves that several $\sigma$ back-donation channels are relevant in these dimers, actually controlling the conformational preference in the $\pi$ adducts. A number of one-electron Beryllium bonds are also studied. Orbital invariant real space arguments clearly show that the role of covalency and charge transfer cannot be ignored.
\end{abstract}

\title{
Introduction
}

The rise in the number of studies regarding non-covalent interactions in this first part of the twentyfirst century is out of discussion. ${ }^{1}$ Once chemists have mastered the art of building individual molecules, it was only a matter of time that the focus turned toward understanding the rules governing supramolecular assemblies. In this soft-matter ${ }^{2}$ regime, it is weak interactions that are responsible for structure and ultimately function in territories as different as biology and crystal engineering. ${ }^{3}$ It comes as no surprise then that, as the number of systems investigated grew, so did the number of specific weak bonds reported. The paradigmatic hydrogen bond (HB) category has thus been enlarged with a large set of new bonding motifs: dihydrogen, halogen, pnicogen, tetrel bonds have been defined and used to control supramolecular structures. And as it happened with the hydrogen bond, ${ }^{4}$ several different, sometimes opposing views about the nature of these interactions have flourished. ${ }^{5-9}$ On one end we find the electrostatic point of view, pioneered by Politzer and Murray. ${ }^{10,11}$ According to this position, it is the electrostatic attraction between an electron depleted region on one fragment, the so-called $\sigma$-hole, and an electron rich one on the other that drives these non-covalent interactions. In this view, which can also be understood as a general- 
ized Lewis acid/base framework or as a weak interaction version of Weinhold's donor-acceptor paradigm, ${ }^{12}$ maps of the electrostatic potential (ESP) play a relevant role in the correct docking of $\sigma$-holes and electron rich domains. On the other hand, many theoretical studies, summarized by the IUPAC ${ }^{13}$ have shown that covalency or dispersion play also a relevant role.

In 2009, following the idea that $\sigma$-holes are indeed playing the role of electron acceptors, the group of Manuel Yáñez and Otilia Mó proposed that Beryllium derivatives, known to be very good Lewis acceptors, should form complexes with Lewis bases. ${ }^{14}$ Since then, these beryllium bonds have been added to the toolkit of new non-covalent links, and have been shown to be considerably strong and dominated by electrostatic interactions. Be-bonds have been found in both $\sigma_{-}{ }^{14}$ and $\pi$-complexes ${ }^{15}$, and their density polarizing abilities have been used to modify the bonding abilities of other moieties, for instance inducing $\sigma$-holes in fluorine-containing systems. ${ }^{16}$ As of today, the nature of the Be-bond has been established by a battery of methods that include standard molecular orbital ideas, ${ }^{17}$ Fock-space energy decomposition analyses like the LMOEDA method, ${ }^{18}$ Weinhold's natural bond orbitals (NBO), ${ }^{12}$ quantum chemical topology (QCT) methods like the quantum theory of atoms in molecules (QTAIM) of Bader and coworkers, ${ }^{19}$ or the electron localization function (ELF) ${ }^{20}$ the natural orbitals for chemical valence of Ziegler, Michalak and Mitoraj $^{21,22}$, etc. Most of these methods have shown that electrostatics is an important player in explaining Be-bonds, but that covalent contributions are not negligible. However, Politzer, Murray and Clark have recently argued strongly against these theoretical constructs. ${ }^{10}$ According to these authors, exchange, Pauli repulsions or orbitals are simply mathematical constructs that are used to obtain an approximate solution of the multi-electron Schrödinger equation. Only electrostatics, via the Hellmann-Feynman theorem, does account for bonding, and other effects, such as charge transfer lie only in the model: the illusion of charge transfer is in the model; the reality of polarization is in the electronic density, the physical observable.

We show here that QCT methods, using quantities which are in principle amenable to experimental determination, provide univocal answers to these questions. By employing reduced density matrices (which are Dirac observables) and QTAIM partitions (which can be and are actually deter- 
mined in experiments) we: (i) gain access to energy components (through the interacting quantum atoms approach, IQA) including electrostatic and covalent terms; (ii) provide effective one-electron pictures that contain correlation effects via natural adaptive orbitals; ${ }^{23}$ and (iii) uncover the distribution of the electron population, thus true charge transfers, with electron distribution functions (EDFs).

As far as we know, this is the first time that Be-bonds are studied using such a combined strategy. We have decided to focus on three sets of aggregates: $\sigma$-complexes, exemplified by the $\mathrm{BeX}_{2}$-LB series, LB being a Lewis base like ammonia or water; $\pi$-complexes, like those formed by $\mathrm{BeX}_{2}$ and ethylene or acetylene; and one-electron bonded complexes, with $\mathrm{LiBe}$ and $\mathrm{BeLiBe}$ as examples. Additional motivations that justify addressing the study of these (and other) beryllium compounds, trying to clarify the main characteristics of their chemical bonds, are purely practical. It is well known, for instance, that beryllium fluoride associates to ADP inhibiting protein ac-

tion. ${ }^{24-30}$ Reactivity patterns of half-sandwich complexes formed by Be are also interesting. ${ }^{31,32}$ Finally, although it is not completely established, it seems that the interaction between $\mathrm{Be}^{2+}$ and several water molecules plays an important role on beryliosis or CBD (chronic beryllium disease), an very often fatal illness caused by this metal. ${ }^{33,34}$ Theoretical analyses of chemical bonds formed by beryllium may help to clarify the possible causes associated to its toxicity. In this work, we focus on the nature of the stabilizing energy components, explaining how electrostatics, but not only electrostatics, accounts for their binding energy. We also consider charge transfer, interpreting it in a crystal clear way as a result of real space resonance of several electron configurations. We start by summarizing the conceptual framework to be used, turning to the computational details and an analysis of our results.

\section{Methodology}

Only Dirac observable densities are used in QCT, that starts with a physical partition of space into chemically meaningful regions. ${ }^{19}$ We use the QTAIM partition, so that $R^{3}=\bigcup_{i}^{m} A_{i}$, where $A_{i}$ is an 
attraction basin of the electron density field, $\rho(\boldsymbol{r})$, usually corresponding to an atom-in-a-molecule. We stress that the atoms of the QTAIM are nowadays obtained routinely both from computations as well as from X-ray diffraction experiments. ${ }^{35}$ Much information can be obtained by examining the local topology of $\rho,{ }^{36}$ but since this QTAIM local operating mode is well known and several works on Be-bonds have used it, we will not consider it in this work. The position space QTAIM partitioning may be directly inherited by all the reduced density matrices (nRDMs) ${ }^{37}$. For instance, the $A_{1} A_{2} \ldots A_{n}$ component of the $n$-th order reduced density (nRD) is simply defined as

$$
\rho_{\mathrm{n}}^{\mathrm{A}_{1} \mathrm{~A}_{2} \ldots \mathrm{A}_{\mathrm{n}}}\left(\mathbf{r}_{1}, \mathbf{r}_{2}, \ldots, \mathbf{r}_{\mathrm{n}}\right)=\rho_{\mathrm{n}}\left(\mathbf{r}_{1} \in \Omega_{\mathrm{A}_{1}}, \mathbf{r}_{2} \in \Omega_{\mathrm{A}_{2}}, \ldots, \mathbf{r}_{\mathrm{n}} \in \Omega_{\mathrm{A}_{\mathrm{n}}}\right) .
$$

Because the electronic energy for a Coulomb Hamiltonian depends only on the 1RDM and the $2 \mathrm{RD}, E=\operatorname{Tr} \hat{h} \rho_{1}\left(\mathbf{r}_{1}, \mathbf{r}_{1}^{\prime}\right)+(1 / 2) \operatorname{Tr} r_{12}^{-1} \rho_{2}\left(\mathbf{r}_{1}, \mathbf{r}_{2}\right)$, we can write $E$ as a sum of one- and two-domain components. This leads to the IQA decomposition,

$$
E=\sum_{\mathrm{A}} E_{\mathrm{self}}^{\mathrm{A}}+\sum_{\mathrm{A}>\mathrm{B}} E_{\mathrm{int}}^{\mathrm{AB}}
$$

In this expression, $E_{\text {self }}^{\mathrm{A}}$ is the self-energy of atom $\mathrm{A}$, which adds all the energy terms that depend only on nuclei (n) and electrons (e) contained in domain A, while the pairwise additive interatomic energy, $E_{\mathrm{int}}^{\mathrm{AB}}$, gathers all contributions containing particles in the $\mathrm{A}$ and $\mathrm{B}$ regions. In this way, $E_{\mathrm{self}}^{\mathrm{A}}=T^{\mathrm{A}}+V_{\mathrm{ne}}^{\mathrm{AA}}+V_{\mathrm{ee}}^{\mathrm{AA}}$ and $E_{\mathrm{int}}^{\mathrm{AB}}=V_{\mathrm{nn}}^{\mathrm{AB}}+V_{\mathrm{ee}}^{\mathrm{AB}}+V_{\mathrm{ne}}^{\mathrm{AB}}+V_{\mathrm{ne}}^{\mathrm{BA}}$, where we have used a clear notation that needs no more comments. If self-energies are measured with respect to given energetic references for each atom or fragment, $E_{0}^{A}$, then deformation energies arise as $E_{\mathrm{def}}^{A}=E_{\mathrm{self}}^{A}-E_{0}^{A}$.

We obtain a fruitful decomposition of $E_{\text {int }}^{\mathrm{AB}}$ if we further partition the 2RD into its Coulomb (J) and exchange-correlation (xc) components, $\rho_{2}\left(\mathbf{r}_{1}, \mathbf{r}_{2}\right)=\rho_{2}^{\mathrm{J}}\left(\mathbf{r}_{1}, \mathbf{r}_{2}\right)+\rho_{2}^{\mathrm{xc}}\left(\mathbf{r}_{1}, \mathbf{r}_{2}\right)$. In this way, we can separate all terms in $E_{\text {int }}$ depending on the one-particle density (that would correspond to the interaction among classical particles) from those calculated from the exchange-correlation density (with no analogue in classical mechanics), so that $E_{\mathrm{int}}^{\mathrm{AB}}=E_{\mathrm{cl}}^{\mathrm{AB}}+E_{\mathrm{xc}}^{\mathrm{AB}}$. The contribution $E_{\mathrm{cl}}^{\mathrm{AB}}$ is thus immediately associated to the electrostatic (ionic) energy component of a chemical bond, tending 
asymptotically to $Q_{\mathrm{A}} Q_{\mathrm{B}} / R_{\mathrm{AB}}$ for charged species, while $E_{\mathrm{xc}}^{\mathrm{AB}}$ i.e., the exchange-correlation energy, represents a measure of covalency ${ }^{38,39}$. Notice that, regarding Politzer et al. considerations, $E_{\mathrm{cl}}$ is the purely classical electrostatic interaction of the modified, mutually polarized, molecular densities.

If not the energy-weighted RDs but the RDs themselves are coarse-grained, we get a general population analysis. ${ }^{37}$ With this, we can decompose general $n$-th order cumulant densities (CDs) $\rho_{n}^{c}\left(\boldsymbol{r}_{1}, \ldots, \boldsymbol{r}_{n}\right)$ that integrate to the total number of electrons $N$ into one-, two- or $n$-center terms: a partition of the $\rho_{c}^{1}=\rho$ provides the standard QTAIM atomic populations, that of $\rho_{2}^{c}=\rho_{\mathrm{xc}}$ gives rise to the well-known localization and delocalization indices which are real space covalent bond orders, and, in general, a decomposition of $\rho_{n}^{c}$ reveals $n$-center bond orders. Doing this with the $N$-th order RD, $\Psi^{*} \Psi$, we can obtain the probability of finding a given number of electrons in each of the $n A$ regions, ${ }^{40-42}$ leading to EDFs. Finally, CDs can be partially coarse-grained, leaving one electron coordinate free of this process that describes a real space natural density of $n$-center bonding. Diagonalizing these densities we get sets of effective one electron functions, the natural adaptive orbitals (NAdOs) ${ }^{23}$ together with their associated natural adaptive occupations. The latter decompose the electron population, the two-center, three-center, etc. bond orders into one-electron components as the order of the cumulant that is diagonalized increases. All these quantities can in principle be obtained from experiments (for instance with X-ray constrained wave functions ${ }^{43,44}$ ).

\section{Computational details}

We have performed IQA decompositions and analyzed EDFs and NAdOs in a set of relevant Bebonded molecules that include the $\mathrm{CO}, \mathrm{H}_{2} \mathrm{O}$ and $\mathrm{NH}_{3}$ complexes of $\mathrm{BeX}_{2}(\mathrm{X}=\mathrm{H}, \mathrm{F}, \mathrm{Cl})$ together with the BeLi, BeLiBe, BeNa, and $\mathrm{C}_{10} \mathrm{H}_{6} \mathrm{Be}_{2} \mathrm{H}_{2}^{-}$systems. All geometry optimizations were carried out at the B3LYP//def2-tzVPD level using the GAMESS package. ${ }^{45}$ Further single points calculations at the B3LYP/def2-qzVPD level using the PySCF suite ${ }^{46}$ were performed for all subsequent analyses, using the scaling approach as described in Ref. 47 For BeLi, BeLiBe, and BeNa 
CASSCF calculations using a aug-cc-pVQZ basis were preferred and obtained also with PySCF. The following notation is used: (electrons,orbitals). The orbital space for each system was selected using the density matrix embedding theory (DMET). ${ }^{48}$ For the bath selection a cutoff of 0.01 was chosen and the following impurities were selected: in the case of BeLi and BeLiBe, the $2 s, 2 p$ orbitals for each atom, resulting in a $(3,10) /(5,15)$ space; for BeNa, the $2 s, 2 p$ orbitals for Be, and the $3 s, 3 p$ for the $\mathrm{Na}$ atom, resulting in a $(3,9)$ space.

IQA integrations were performed using $\beta$-spheres with radii between 0.1 and 0.3 bohr. Restricted angular Lebedev quadratures with 5810 points and 451 points Gauss-Chebyshev mapped radial grids were used inside the $\beta$-spheres, with $L$ expansions cut at $l=10$. Outside the $\beta$-spheres, extended 5810-point Lebedev, 551- and 651- mapped radial point Gauss-Legendre quadratures, and $L$ expansions up to $l=12$ were selected. Total energies reconstructed from these IQA decompositions differ in less than $1.0 \mathrm{kcal} / \mathrm{mol}$ from those of the parent electronic structure codes, and since error cancelation does not occur in these numerical integrations, each of the computed interactions is considerably more accurate than this figure. This accuracy is enough for the aims of this paper. All IQA calculations were done with our in-house code PROMOLDEN, available upon request $^{49}$. Electron distribution functions (EDF) were obtained using our in house EDF code, ${ }^{50}$ and natural adaptive orbitals (NAdOs) with DENMAT. ${ }^{51}$ In both cases the atomic overlap matrices (AOM) that are needed to feed these codes were obtained from PROMOLDEN.

\section{Beryllium $\sigma$-complexes}

The first Beryllium bonds analyzed ${ }^{14}$ were $\sigma$ complexes between $\mathrm{BeX}_{2}(\mathrm{X}=\mathrm{H}, \mathrm{F}, \mathrm{Cl})$ units and several Lewis bases like water or ammonia. Besides recognizing the important geometrical distortions suffered by the linear $\mathrm{BeX}_{2}$ moiety upon complexation as well as systematizing the considerable strength of the interactions, their chemical bonding was interpreted in terms of local topological descriptors coming from the electron density or the ELF and via the natural bond orbital (NBO) analysis. This led to propose an important role of the LB lone pairs as donors to both the empty 
$p$ orbital of $\mathrm{Be}$ and to the $\sigma_{\mathrm{BeX}}^{*}$ antibond. Further analyses ${ }^{52}$ have even reported some IQA data, but have failed, in our opinion, to provide much more than a description of the computed data. As we expect to show, charge transfer between the units is an important player that helps rationalizing conformer preference, leading to both $\mathrm{LB} \rightarrow \mathrm{BeX}_{2}$ donation as well as to $\mathrm{LB} \leftarrow \mathrm{BeX}_{2}$ back-donation channels which as far as we know have not been reported.

Table 1: Several IQA properties for $\mathrm{BeX}_{2}$-Lewis base (LB) complexes. A and B denote $\mathrm{BeX}_{2}$ and $L B$, respectively. $E_{\mathrm{def}}^{A}$ and $E_{\mathrm{def}}^{B}$ are given with respect to the total energies of the isolated fragments at the geometry they have in the complex, $E_{\text {bind }}^{\mathrm{AB}}=E_{\mathrm{int}}^{\mathrm{AB}}+E_{\mathrm{def}}^{\mathrm{A}}+E_{\mathrm{def}}^{\mathrm{B}}$, and $E_{\mathrm{dis}}^{\mathrm{AB}}=$ $E_{\text {bind }}^{\mathrm{AB}}+E_{\text {relax }}$, where $E_{\text {relax }}$ is the energy associated to the geometric relaxation of the fragments to their own optimal geometries. Energies in kcal/mol, $Q_{A}$ in electrons and $\delta_{A B}$ in electron pairs.

\begin{tabular}{|c|c|c|c|c|c|c|c|c|c|}
\hline System & $E_{\mathrm{int}}^{\mathrm{AB}}$ & $E_{\mathrm{cl}}^{\mathrm{AB}}$ & $E_{\mathrm{xc}}^{\mathrm{AB}}$ & $E_{\mathrm{def}}^{\mathrm{A}}$ & $E_{\mathrm{def}}^{\mathrm{B}}$ & $E_{\text {bind }}^{\mathrm{AB}}$ & $E_{\mathrm{dis}}^{\mathrm{AB}}$ & $Q_{\mathrm{A}}$ & $\delta^{\mathrm{AB}}$ \\
\hline $\mathrm{H}_{2} \mathrm{Be} \cdots \mathrm{OH}_{2}$ & -111.03 & -54.30 & -56.73 & 35.27 & 50.45 & 25.31 & -18.29 & 0.014 & 0.467 \\
\hline $\mathrm{H}_{2} \mathrm{Be} \cdots \mathrm{NH}_{3}$ & -118.76 & -60.89 & -57.88 & 34.17 & 52.45 & -32.15 & -22.70 & -0.010 & 0.481 \\
\hline $\mathrm{H}_{2} \mathrm{Be} \cdots \mathrm{CO}$ & -103.18 & -38.16 & -65.02 & 44.94 & 45.90 & -12.34 & -6.24 & 0.076 & 0.593 \\
\hline $\mathrm{H}_{2} \mathrm{Be} \cdots \mathrm{C}_{2} \mathrm{H}_{4}{ }^{a}$ & -119.53 & -39.71 & -79.82 & 52.67 & 51.48 & -15.38 & -5.67 & 0.080 & 0.755 \\
\hline $\mathrm{H}_{2} \mathrm{Be} \cdots \mathrm{C}_{2} \mathrm{H}_{4} b$ & -36.14 & -7.42 & -28.73 & 10.81 & 21.95 & -3.39 & -1.71 & -0.030 & 0.311 \\
\hline $\mathrm{H}_{2} \mathrm{Be} \cdots \mathrm{C}_{2} \mathrm{H}_{2}{ }^{a}$ & -139.34 & -49.00 & -90.34 & 60.25 & 59.51 & -19.57 & -8.85 & 0.103 & 0.826 \\
\hline $\mathrm{H}_{2} \mathrm{Be} \cdots \mathrm{C}_{2} \mathrm{H}_{2}{ }^{b}$ & -15.41 & -1.84 & -13.57 & 5.32 & 9.48 & -0.62 & -0.51 & -0.014 & 0.162 \\
\hline $\mathrm{F}_{2} \mathrm{Be} \cdots \mathrm{OH}_{2}$ & -102.09 & -52.19 & -49.90 & 27.35 & 44.99 & -29.75 & -20.41 & -0.024 & 0.387 \\
\hline $\mathrm{F}_{2} \mathrm{Be} \cdots \mathrm{NH}_{3}$ & 46 & $-5 c$ & -53.51 & 26.83 & $4 \varepsilon$ & -37.79 & -26.32 & 45 & 0.422 \\
\hline $\mathrm{F}_{2} \mathrm{Be} \cdots \mathrm{CO}$ & -66.34 & -23.72 & -42.62 & 20.90 & 34.61 & -10.83 & -5.37 & -0.020 & 0.372 \\
\hline $\mathrm{F}_{2} \mathrm{Be} \cdots \mathrm{C}_{2} \mathrm{H}_{4}{ }^{a}$ & -57.40 & -16.33 & -41.07 & 16.84 & 30.94 & -9.62 & -3.91 & -0.047 & 0.399 \\
\hline $\mathrm{F}_{2} \mathrm{Be} \cdots \mathrm{C}_{2} \mathrm{H}_{4} b$ & -60.32 & -19.17 & 15 & 16.21 & 32.67 & -11.44 & -5.10 & -0.050 & 0.394 \\
\hline $\mathrm{F}_{2} \mathrm{Be} \cdots \mathrm{C}_{2} \mathrm{H}_{2}{ }^{a}$ & -65.59 & -21.35 & -44.24 & 19.55 & 34.17 & -11.87 & -5.90 & -0.041 & 0.416 \\
\hline $\mathrm{F}_{2} \mathrm{Be} \cdots \mathrm{C}_{2} \mathrm{H}_{2}{ }^{b}$ & -50.13 & -14.48 & -35.64 & 13.54 & 28.48 & -8.11 & -2.91 & -0.045 & 0.346 \\
\hline $\mathrm{Cl}_{2} \mathrm{Be} \cdot \cdots \mathrm{OH}_{2}$ & -127.37 & -67.44 & -59.93 & 39.26 & 55.75 & -32.35 & -21.12 & -0.001 & 0.502 \\
\hline $\mathrm{Cl}_{2} \mathrm{Be} \cdots \mathrm{NH}_{3}$ & -137.32 & -74.89 & -62.43 & 37.21 & 59.54 & -40.56 & -27.77 & -0.027 & 0.527 \\
\hline $\mathrm{Cl}_{2} \mathrm{Be} \cdots \mathrm{CO}$ & -100.40 & -40.29 & -60.11 & 39.47 & 47.78 & -13.15 & -5.11 & 0.029 & 0.563 \\
\hline $\mathrm{Cl}_{2} \mathrm{Be} \cdot \mathrm{C}_{2} \mathrm{H}_{4}{ }^{a}$ & -78.91 & -24.96 & -53.95 & 25.53 & 42.44 & -10.94 & -1.13 & -0.028 & 0.557 \\
\hline $\mathrm{Cl}_{2} \mathrm{Be} \cdots \mathrm{C}_{2} \mathrm{H}_{4}{ }^{b}$ & -76.68 & -25.83 & -50.85 & 22.43 & 42.41 & -11.84 & -2.94 & -0.037 & 0.521 \\
\hline $\mathrm{Cl}_{2} \mathrm{Be} \cdots \mathrm{C}_{2} \mathrm{H}_{2}{ }^{a}$ & -93.21 & -33.89 & -59.32 & 31.52 & 47.64 & -14.05 & -3.04 & -0.017 & 0.590 \\
\hline $\mathrm{Cl}_{2} \mathrm{Be} \cdots \mathrm{C}_{2} \mathrm{H}_{2}{ }^{b}$ & -64.45 & -20.29 & -44.16 & 19.98 & 36.77 & -7.70 & -0.70 & -0.035 & 0.457 \\
\hline
\end{tabular}

In order to keep the discussion succinct, we have only considered the $\mathrm{H}_{2} \mathrm{O}, \mathrm{NH}_{3}$, and $\mathrm{CO}$ dimers with $\mathrm{BeX}_{2}(\mathrm{X}=\mathrm{H}, \mathrm{F}, \mathrm{Cl})$. In all cases the $\mathrm{X}-\mathrm{Be}-\mathrm{X}$ angle differs considerably from $180^{\circ}$, see the supplementary information. Relevant IQA data are reported on Table 1. As it is well known, all $\mathrm{BeX}_{2}$ systems are considerably ionic with QTAIM charges of about 1.7 and -0.85 electrons for the 
$\mathrm{Be}$ and $\mathrm{X}$ atoms respectively. In all the cases the $\mathrm{L}$ shell of Beryllium has been transferred to the $\mathrm{X}$ companion, as seen by the laplacian. Complexation polarizes the $\mathrm{BeX}_{2}$ fragment, that gets bent leaving a large positive laplacian region toward which the lone pair of the LB points. All Be $\cdots$ LB bond critical points display positive laplacian. It is interesting to notice that the $\mathrm{BeX}_{2} \cdots \mathrm{H}_{2} \mathrm{O}$ systems are planar, with a $\sigma+\pi$ distribution of the oxygen's lone pairs adequate for $\pi$ donation, as suggested by NBO analyses.

Total charge transfer between the fragments is in general larger than in typical hydrogen bonded (HB) complexes, ${ }^{53}$ getting as large as 0.08 electrons in $\mathrm{BeH}_{2} \cdots \mathrm{CO}$. Notice that the direction of charge transfer oscillates. A negatively charged LB necessarily implies that there exist other delocalization channels beside the $l p \rightarrow p_{\mathrm{Be}}$ or $l p \rightarrow \sigma_{\mathrm{BeX}}^{*}$. This back-donation dominates effectively in the $\mathrm{BeH}_{2}$ complexes with water and in $\mathrm{BeX}_{2} \cdots \mathrm{CO}$ with $\mathrm{X}=\mathrm{H}, \mathrm{Cl}$. We will return to the origin of this interesting observation.

As energy components are regarded, both electrostatic and covalent contributions are important in the stabilization of the complexes. In all the $\sigma$ dimers it is the deformation energy of the Lewis base that dominates, as it is also the case in HB dimers. However, deformations in the present Be-bonds are several times larger than the ones found in HBs. The deformation of the LB in the water dimer, for instance, is only about $8 \mathrm{kcal} / \mathrm{mol}$, to be compared with $50 \mathrm{kcal} / \mathrm{mol}$ in the $\mathrm{BeH}_{2}$ complex. The distortions induced by mutual polarization of the fragments in Beryllium bonds seem to be rather intense.

Electron delocalization is extremely relevant. The A-B delocalization indices lie around $0.3-$ 0.6, being much larger than in HBs (one of the largest is found in the strongly bound $\mathrm{FHF}^{-}$system, with a $\delta$ close to $0.2 .{ }^{53}$ ). This makes the stronger nature of distortions and interactions in Be-bonds clear as compared to HBs. As in usual (relatively) weak complexes, the sum of the fragments' deformation energies and $E_{\mathrm{xc}}^{\mathrm{AB}}$, which we have related several times to the exchange-repulsion terms in other approaches, ${ }^{53,54}$ is positive, again larger (by one order of magnitude) than the values found in HBs. Delocalization thus does not compensate deformation. This behavior is typical of traditional charge transfer complexes or very ionic bonds, being in favor of electrostatic bonding 
models. But, as we have also explained before, ${ }^{53}$ covalency cannot be disregarded, since $E_{\mathrm{cl}}^{\mathrm{AB}}$ is not stabilizing enough as to overcome deformations.

Table 2: Electron Distribution Functions (EDF) for some $\mathrm{BeX}_{2}$-Lewis base (LB) complexes. $A$ and $B$ denote $B e X_{2}$ and $L B$, respectively. $p\left(B_{2}\right)$ denotes the probability of the nominal RSRS and $p\left(\operatorname{BeX}_{2}^{q}\right)$ the probability of the RSRS of the fragment $\operatorname{BeX}_{2}$ with a total charge $q$.

\begin{tabular}{|l|rrrrr|}
\hline System & $p\left(\mathrm{BeX}_{2}\right)$ & $p\left(\mathrm{BeX}_{2}^{-}\right)$ & $p\left(\mathrm{BeX}_{2}^{+}\right)$ & $p\left(\mathrm{BeX}_{2}^{2-}\right)$ & $p\left(\mathrm{BeX}_{2}^{2+}\right)$ \\
\hline $\mathrm{H}_{2} \mathrm{Be} \cdots \mathrm{OH}_{2}$ & 0.795 & 0.092 & 0.103 & 0.004 & 0.005 \\
$\mathrm{H}_{2} \mathrm{Be} \cdots \mathrm{NH}_{3}$ & 0.788 & 0.106 & 0.096 & 0.004 & 0.004 \\
$\mathrm{H}_{2} \mathrm{Be} \cdots \mathrm{CO}$ & 0.741 & 0.093 & 0.153 & 0.003 & 0.011 \\
$\mathrm{H}_{2} \mathrm{Be} \cdots \mathrm{C}_{2} \mathrm{H}_{4}{ }^{\mathrm{a}}$ & 0.684 & 0.115 & 0.177 & 0.007 & 0.015 \\
$\mathrm{H}_{2} \mathrm{Be} \cdots \mathrm{C}_{2} \mathrm{H}_{4}{ }^{\mathrm{b}}$ & 0.855 & 0.084 & 0.057 & 0.003 & 0.001 \\
$\mathrm{H}_{2} \mathrm{Be} \cdots \mathrm{C}_{2} \mathrm{H}_{2}{ }^{\mathrm{a}}$ & 0.662 & 0.116 & 0.195 & 0.007 & 0.019 \\
$\mathrm{H}_{2} \mathrm{Be} \cdots \mathrm{C}_{2} \mathrm{H}_{2}{ }^{\mathrm{b}}$ & 0.922 & 0.045 & 0.032 & 0.001 & 0.000 \\
\hline $\mathrm{F}_{2} \mathrm{Be} \cdots \mathrm{OH}_{2}$ & 0.826 & 0.094 & 0.073 & 0.004 & 0.003 \\
$\mathrm{~F}_{2} \mathrm{Be} \cdots \mathrm{NH}_{3}$ & 0.811 & 0.111 & 0.070 & 0.005 & 0.003 \\
$\mathrm{~F}_{2} \mathrm{Be} \cdots \mathrm{CO}$ & 0.830 & 0.092 & 0.072 & 0.003 & 0.003 \\
$\mathrm{~F}_{2} \mathrm{Be} \cdots \mathrm{C}_{2} \mathrm{H}_{4}{ }^{\mathrm{a}}$ & 0.820 & 0.108 & 0.065 & 0.005 & 0.002 \\
$\mathrm{~F}_{2} \mathrm{Be} \cdots \mathrm{C}_{2} \mathrm{H}_{4}{ }^{\mathrm{b}}$ & 0.822 & 0.108 & 0.063 & 0.005 & 0.002 \\
$\mathrm{~F}_{2} \mathrm{Be} \cdots \mathrm{C}_{2} \mathrm{H}_{2}{ }^{\mathrm{a}}$ & 0.815 & 0.107 & 0.071 & 0.005 & 0.003 \\
$\mathrm{~F}_{2} \mathrm{Be} \cdots \mathrm{C}_{2} \mathrm{H}_{2}{ }^{\mathrm{b}}$ & 0.842 & 0.097 & 0.056 & 0.004 & 0.002 \\
\hline $\mathrm{Cl}_{2} \mathrm{Be} \cdots \mathrm{OH}_{2}$ & 0.784 & 0.103 & 0.102 & 0.005 & 0.006 \\
$\mathrm{Cl}_{2} \mathrm{Be} \cdots \mathrm{NH}_{3}$ & 0.772 & 0.121 & 0.095 & 0.006 & 0.005 \\
$\mathrm{Cl}_{2} \mathrm{Be} \cdots \mathrm{CO}$ & 0.758 & 0.125 & 0.104 & 0.004 & 0.009 \\
$\mathrm{Cl}_{2} \mathrm{Be} \cdots \mathrm{C}_{2} \mathrm{H}_{4}{ }^{\mathrm{a}}$ & 0.763 & 0.125 & 0.100 & 0.008 & 0.013 \\
$\mathrm{Cl}_{2} \mathrm{Be} \cdots \mathrm{C}_{2} \mathrm{H}_{4}{ }^{\mathrm{b}}$ & 0.775 & 0.123 & 0.090 & 0.015 & 0.025 \\
$\mathrm{Cl}_{2} \mathrm{Be} \cdots \mathrm{C}_{2} \mathrm{H}_{2}{ }^{\mathrm{a}}$ & 0.752 & 0.124 & 0.109 & 0.007 & 0.007 \\
$\mathrm{Cl}_{2} \mathrm{Be} \cdots \mathrm{C}_{2} \mathrm{H}_{2}{ }^{\mathrm{b}}$ & 0.800 & 0.111 & 0.080 & 0.005 & 0.003 \\
\hline
\end{tabular}

The nature of charge transfer deserves specific consideration. Table 2 contains the most representative real space resonance structures (RSRSs) contributing to our Beryllium complexes. Notice that the overall inter-fragment charge transfer sign comes from the relative weight of the $\mathrm{BeX}_{2}^{-}$ versus the $\mathrm{BeX}_{2}^{+}$structures, given the small values of more highly charged structures $\mathrm{BeX}_{2}^{2-}$ and $\mathrm{BeX}_{2}^{2+}$. The non-negligible probabilities of both the $\mathrm{BeX}_{2}^{-}$and $\mathrm{BeX}_{2}^{+}$RSRSs point towards polarized symmetric (thus covalent-like) delocalizations or to several independent charge transfer channels. We can distinguish between both possibilities by exploring the two-fragment natural 
adaptive orbitals that decompose the overall delocalization index into effective one-electron components. Table 3 reports the most relevant contributions for each of the complexes, while Fig. 1 provides pictorial images in the $\mathrm{BeH}_{2} \cdots \mathrm{H}_{2} \mathrm{O}$ and $\mathrm{BeF}_{2} \cdots \mathrm{H}_{2} \mathrm{O}$ cases. There are several interesting points that deserve being discussed.

Table 3: Contributions to $\delta^{\mathrm{AB}}$ greater than 0.01 of the two-fragment NAdOs of $\mathrm{BeX}_{2}$-Lewis base (LB) systems. Each number represents the occupation of a natural adaptive orbital (NAdO). See Figure 1 for the cases of $\mathrm{BeH}_{2} \cdots \mathrm{H}_{2} \mathrm{O}$ and $\mathrm{BeF}_{2} \cdots \mathrm{H}_{2} \mathrm{O}$.

\begin{tabular}{|l|ccc|ccc|ccc|}
\hline $\mathrm{BeX}_{2} \rightarrow$ & \multicolumn{3}{|c|}{$\mathrm{BeH}_{2}$} & \multicolumn{3}{c|}{$\mathrm{BeF}_{2}$} & \multicolumn{3}{c|}{$\mathrm{BeCl}_{2}$} \\
\hline $\mathrm{LB} \rightarrow$ & $\mathrm{H}_{2} \mathrm{O}$ & $\mathrm{NH}_{3}$ & $\mathrm{CO}$ & $\mathrm{H}_{2} \mathrm{O}$ & $\mathrm{NH}_{3}$ & $\mathrm{CO}$ & $\mathrm{H}_{2} \mathrm{O}$ & $\mathrm{NH}_{3}$ & $\mathrm{CO}$ \\
\hline & 0.154 & 0.213 & 0.287 & 0.155 & 0.214 & 0.194 & 0.169 & 0.234 & 0.233 \\
& 0.121 & 0.126 & 0.217 & 0.066 & 0.056 & 0.066 & 0.103 & 0.084 & 0.151 \\
& 0.119 & 0.100 & 0.075 & 0.054 & 0.056 & 0.053 & 0.080 & 0.081 & 0.074 \\
& 0.048 & 0.018 & & 0.038 & 0.035 & 0.036 & 0.051 & 0.043 & 0.065 \\
& 0.017 & 0.016 & & 0.030 & 0.023 & & 0.035 & 0.030 & \\
& & & & 0.020 & 0.014 & & 0.027 & 0.019 & \\
& & & & & & & 0.010 & 0.011 & \\
& & & & & & & 0.010 & & \\
\hline
\end{tabular}

Firstly, the dominant channel is always that involving the base $\sigma$ lone pair. Its intensity is largest in the ammonia complexes, as expected, if we exclude the $\mathrm{BeH}_{2} \cdots \mathrm{CO}$ system. In the second place, the LB to $p_{\mathrm{Be}}$ donation, the fourth component in both rows of Fig. 1, is never second in relevance, as NBO arguments suggest. Contrarily, its donating capacity is relatively low. This type of discrepancies between NBO and real space considerations is rather usual, stemming from the NBO's use of localized basis functions in a second order perturbation expansion. More interesting are the $\mathrm{BeX}_{2}$ to LB back-donation channels. They delocalize charge directly onto the base, and are formed by the symmetric and antisymmetric combination of $\sigma$-like functions mainly localized on the $\mathrm{X}_{2}$ moieties. Turning to a localized NAdOs scheme, if we would like to, they are simply the two $\sigma$ Be-X bonds. We have found that the $\sigma$ back-donation capacity of these channels is largest in $\mathrm{BeH}_{2}$, with easily polarizable hydride-like entities. It decreases considerably in $\mathrm{BeF}_{2}$, where the NAdOs are combinations of highly localized non-bonding $p$ orbitals of the $\mathrm{F}$ atoms, and it increases again as the fluorines are substituted by more diffuse and polarizable $\mathrm{Cl}$ substituents. The relevance of the $\sigma$ back-donation we are describing in the chemistry of these compounds 
remains to be examined. To check the generality of the arguments here presented, we have also computed conformations of the $\sigma \mathrm{H}_{2} \mathrm{O}$ complexes where the $\mathrm{BeX}_{2}$ molecular plane is orthogonal to that of the $\mathrm{H}_{2} \mathrm{O}$ molecule. The importance of the back-donation decreases considerably, as expected. In the $\mathrm{BeH}_{2}$ case, for instance, the effect is so large that the $\mathrm{BeH}_{2}$ fragment changes polarity, with a net charge of -0.005 a.u.

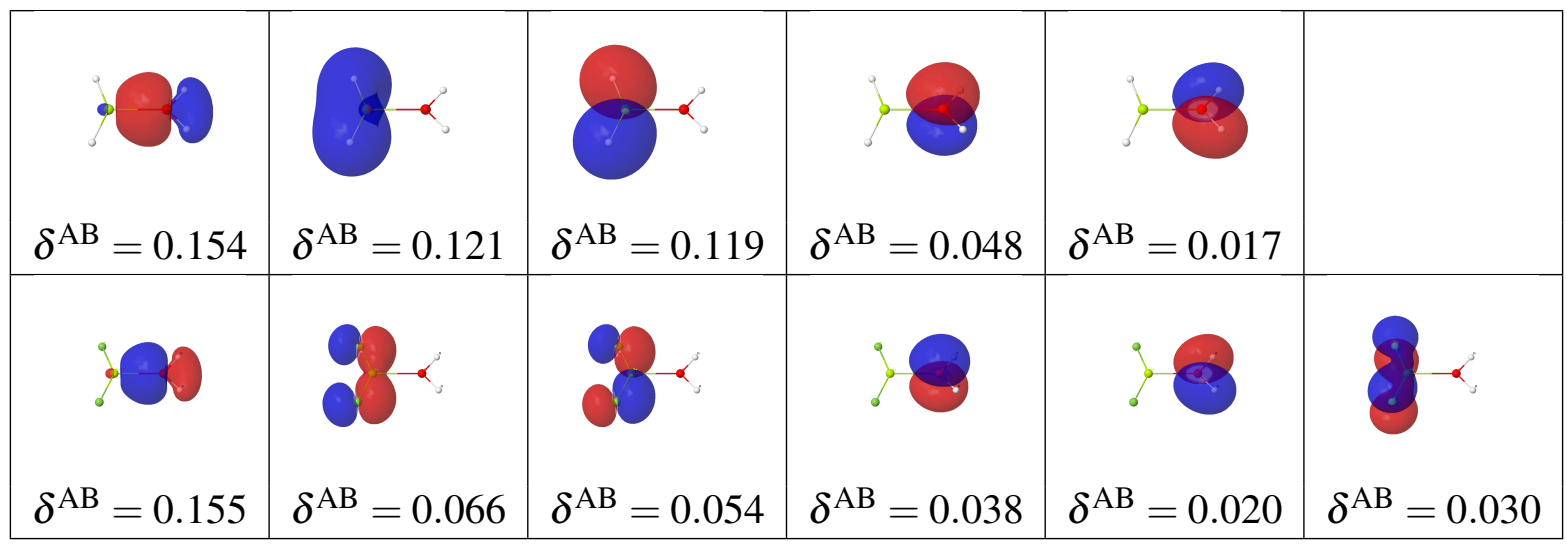

Figure 1: The most highly occupied NAdOs of the $\mathrm{BeH}_{2} \cdots \mathrm{H}_{2} \mathrm{O}$ (top) and $\mathrm{BeF}_{2} \cdots \mathrm{H}_{2} \mathrm{O}$ (bottom) systems. The isosurface shown corresponds to $|\phi|=0.05$ a.u. Their contribution to the $\delta^{\mathrm{AB}}$ delocalization index are shown below.

It is easy to check that a reasonable estimate of the total charge transfer can be obtained by: (i) classifying the NAdO channels into those basically localized in the $\mathrm{BeX}_{2}$ or LB fragments; (ii) adding the occupation numbers of both classes and, finally; (iii) subtracting them to get a grand total. This allows to rationalize easily the oscillating net charge pattern of the complexes found in Table 1 and to construct a donating and back-donating scale of the $\mathrm{LB}$ and $\mathrm{BeX}_{2}$ moieties. For the former $\mathrm{CO} \approx \mathrm{NH}_{3}>\mathrm{H}_{2} \mathrm{O}$, for the latter, $\mathrm{BeH}_{2}>\mathrm{BeCl}_{2}>\mathrm{BeF}_{2}$.

\section{$\pi$-bonded complexes}

Soon after the $\mathrm{BeX}_{2} \sigma$ complexes were examined it was found that the $\mathrm{BeX}_{2}$ species would also embark on $\pi$ bonding with molecules such as ethylene or acetylene. Although weaker in general than the previously analyzed $\sigma$ dimers, these $\pi$ complexes have binding energies typical of hydrogen bonded systems, displaying QTAIM critical points characteristic of $\pi$ interactions and 
being described from the NBO perspective through equivalent $\pi \rightarrow p_{\mathrm{Be}}$ and $\pi \rightarrow \sigma_{\mathrm{BeX}}^{*}$ donoracceptor contributions. An interesting point that has not been thoroughly investigated is the origin of the preference between parallel/perpendicular conformations: $\mathrm{BeF}_{2}$ and $\mathrm{BeCl}_{2}$ prefer to locate their molecular planes coinciding with the plane that contains the C-C internuclear axis [parallel conformation, (a)] in acetylene complexes, but orthogonal to it [orthogonal conformation, (b)] in ethylene ones, while $\mathrm{BeH}_{2}$ prefers the parallel conformation in both cases, the difference in energy between the two conformers being rather large in this last case. We have thus investigated both conformations and shown our IQA results also in Table 1.

A first insight is related to the net charge transfer in the dimers. In the $\mathrm{BeH}_{2}$ case the most stable conformers are those in which this moiety is positively charged. In fact, the perpendicular conformers display much smaller charges (and of opposite signs). All IQA energetic indicators point toward a much stronger interaction (both electrostatic and covalent) as well as larger deformation energies in the (a) conformations. It is also interesting to notice that in these (a) isomers it is the $\mathrm{BeH}_{2}$ moiety which is mostly deformed. This tells about the role of the hydrogens in the stabilization of the complex, leading again to backdonation issues.

Table 4: Contributions to $\delta^{\mathrm{AB}}$ greater than 0.01 of the two-fragment NAdOs of $\mathrm{BeX}_{2} \cdots \mathrm{C}_{2} \mathbf{H}_{4}^{a, b}$ and $\mathrm{BeX}_{2}-\mathrm{C}_{2} \mathrm{H}_{2}^{a, b}(\mathrm{X}=\mathbf{H}, \mathbf{F})$ systems. Each number represents the occupation of a natural adaptive orbital (NAdO). See Figure 2 for the cases $\mathrm{BeH}_{2} \cdots \mathrm{C}_{2} \mathrm{H}_{4}^{a, b}$ and $\mathbf{B e H}_{2} \cdots \mathbf{C}_{2} \mathbf{H}_{2}^{a, b}$.

\begin{tabular}{|cccc|cccc|cccc|}
\hline \multicolumn{5}{|c|}{$\mathrm{BeH}_{2}$} & \multicolumn{5}{c|}{$\mathrm{BeF}_{2}$} & \multicolumn{4}{c|}{$\mathrm{BeCl}_{2}$} \\
\hline $\mathrm{C}_{2} \mathrm{H}_{4}^{a}$ & $\mathrm{C}_{2} \mathrm{H}_{4}^{b}$ & $\mathrm{C}_{2} \mathrm{H}_{2}^{a}$ & $\mathrm{C}_{2} \mathrm{H}_{2}^{b}$ & $\mathrm{C}_{2} \mathrm{H}_{4}^{a}$ & $\mathrm{C}_{2} \mathrm{H}_{4}^{b}$ & $\mathrm{C}_{2} \mathrm{H}_{2}^{a}$ & $\mathrm{C}_{2} \mathrm{H}_{2}^{b}$ & $\mathrm{C}_{2} \mathrm{H}_{4}^{a}$ & $\mathrm{C}_{2} \mathrm{H}_{4}^{b}$ & $\mathrm{C}_{2} \mathrm{H}_{2}^{a}$ & $\mathrm{C}_{2} \mathrm{H}_{2}^{b}$ \\
\hline 0.348 & 0.158 & 0.393 & 0.079 & 0.197 & 0.199 & 0.186 & 0.170 & 0.234 & 0.229 & 0.222 & 0.198 \\
0.248 & 0.080 & 0.236 & 0.048 & 0.060 & 0.057 & 0.066 & 0.057 & 0.116 & 0.082 & 0.129 & 0.083 \\
0.093 & 0.047 & 0.099 & 0.019 & 0.057 & 0.040 & 0.060 & 0.033 & 0.080 & 0.063 & 0.082 & 0.049 \\
& & 0.064 & & 0.021 & 0.036 & 0.028 & 0.032 & 0.030 & 0.058 & 0.041 & 0.045 \\
& & & & & & 0.021 & 0.029 & 0.010 & 0.024 & 0.031 & 0.045 \\
\hline
\end{tabular}

When $\mathrm{X}=\mathrm{F}, \mathrm{Cl}$, the situation changes. Now the hydrocarbon donation channels dominate, and in every case the net charge of the $\mathrm{BeX}_{2}$ species is negative. The ability of the $p$-like lone pairs of $\mathrm{X}$ to participate in bonding interactions in both conformations makes the balance subtle with ethylene, while the conformer discrimination is more neat with acetylene. In all the conformers 
$E_{\mathrm{def}}$ of the $\mathrm{BeX}_{2}$ fragment is considerably smaller than that of the hydrocarbon. For the $\mathrm{BeCl}_{2}$ ethylenic complex an interesting situation arises. The (b) conformer has a smaller total interaction energy than the (a) one, although it is the most stable of the two. Actually, the final energetic difference is close to that in electrostatic interactions, so that deformation energies and covalent contributions cancel their difference for both conformers.

Fig. 2 shows the most relevant NAdOs and their contribution to the inter-fragment delocalization indices for the parallel and orthogonal conformers of $\mathrm{BeH}_{2}$ with ethylene and acetylene. The NAdOs occupation numbers for the three complexes $\mathrm{BeX}_{2}(\mathrm{X}=\mathrm{H}, \mathrm{F}, \mathrm{Cl})$ are found in Table 4 . It is obvious that the (b) conformations quench the $\sigma$ back-donation channels from the hydrides to the carbon atoms, which are dominant in the parallel ones and explain the charge transfer observed. Without these channels, the $\mathrm{H}-\mathrm{Be}-\mathrm{H}$ angle is considerably closer to $180^{\circ}$, and the $\mathrm{Be}-\mathrm{C}$ distance increases. As a result, also the $\pi \rightarrow \mathrm{BeH}_{2}$ donation decreases in strength.

$\mathrm{BeX}_{2}$ with $\mathrm{X}=\mathrm{F}, \mathrm{Cl}$ behave differently thanks to the $p$-symmetry orbitals of the halogen. We only analyze in detail the F complexes, since the conclusions are similar for $\mathrm{X}=\mathrm{Cl}$. Fig. 3 collects the relevant NAdOs. The ethylene compounds display four basic delocalization channels. The most intense one (i) is the $\pi_{\mathrm{C}_{2} \mathrm{H}_{4}} \rightarrow \mathrm{BeF}_{2}$ that accounts for the angularization of $\mathrm{BeF}_{2}$. The rest are $\mathrm{BeF}_{2} \rightarrow \mathrm{C}_{2} \mathrm{H}_{4}$ back-donation terms that involve the symmetric and antisymmetric combination of F $p$ non-bonding orbitals, i.e. two localized $p$ non-bonding orbitals (iia, iib), plus the symmetric $\pi$-symmetry bonding orbital formed by the in-phase combination of the three $p_{z}$ functions corresponding to the F-Be-F atoms (iii). The balance determining the stability of the (a) and (b) conformers is subtle. In the orthogonal one (b, the most stable) and in agreement with intuition, channel (iii) becomes reinforced, while channels (iia, iib) weaken.

Finally, five channels become relevant in the acetylenic compounds. Fig. 3 shows that now a second $\mathrm{C}_{2} \mathrm{H}_{2} \rightarrow \mathrm{BeF}_{2}$ component in $\pi$ conformation becomes relevant, but that all the rest contributions are reinforced by symmetry constraints in the parallel conformer.

We thus come to the conclusion that the combination of IQA with the NAdOs decomposition offers a consistent image of $\sigma$ and $\pi \mathrm{BeX}_{2}$ Beryllium bonds, in which electrostatic interactions 


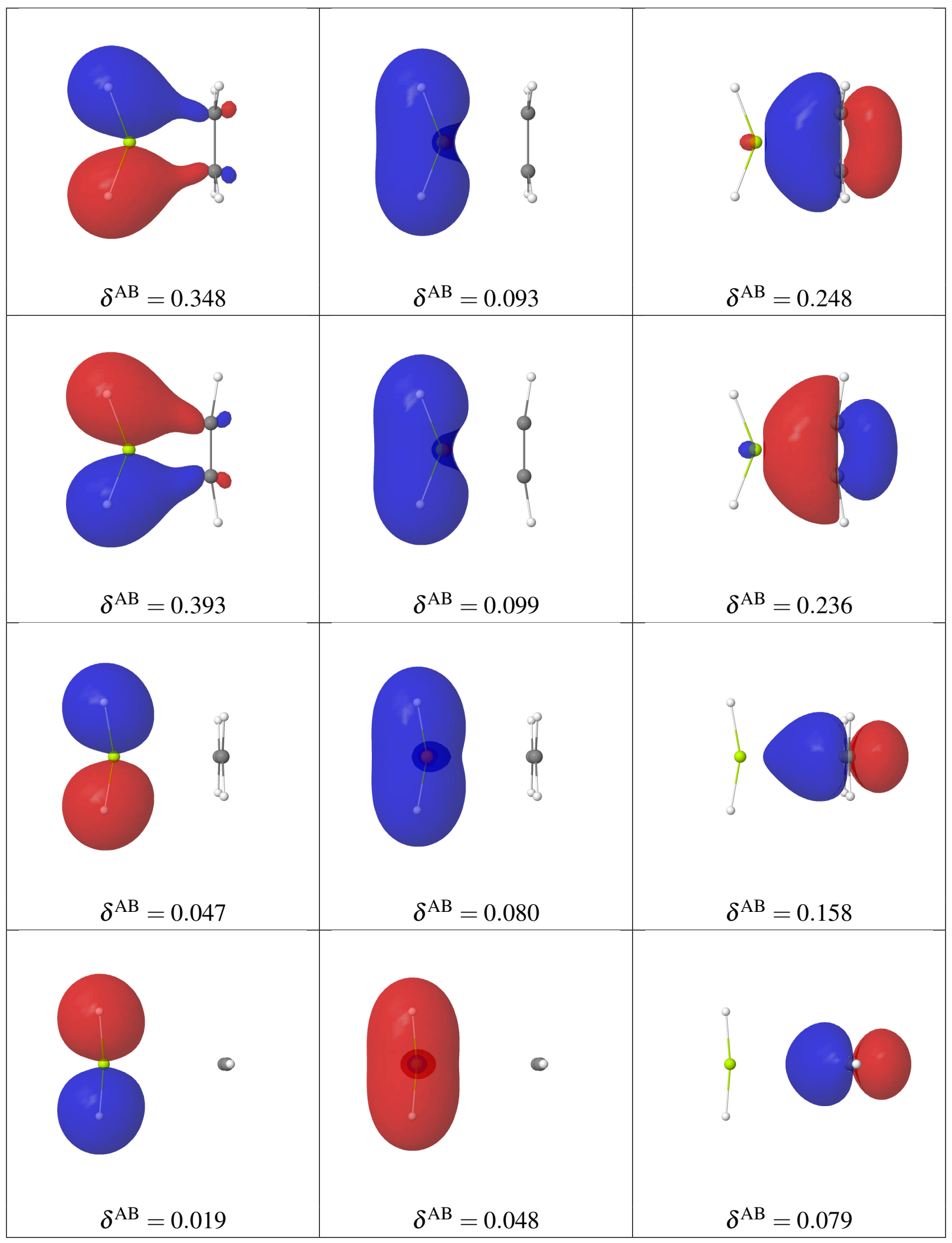

Figure 2: The three most highly occupied NAdOs of the $\mathrm{BeH}_{2} \cdots$ LB systems, with $\mathrm{LB}=\mathrm{C}_{2} \mathrm{H}_{4}{ }^{a}$ ( $1^{\text {st }}$ row), $\mathrm{C}_{2} \mathrm{H}_{2}{ }^{a}$ (2 ${ }^{\text {nd }}$ row), $\mathrm{C}_{2} \mathrm{H}_{4}{ }^{b}$ ( $3^{\text {rd }}$ row), and $\mathrm{C}_{2} \mathrm{H}_{2}{ }^{b}$ ( $4^{\text {th }}$ row). The isosurface shown corresponds to $|\phi|=0.05$ a.u. Their contribution to the $\delta^{\mathrm{AB}}$ delocalization index are shown below. 


\begin{tabular}{|c|c|c|c|c|}
\hline$\delta^{\mathrm{AB}}=0.197$ & $\delta^{\mathrm{AB}}=0.057$ & $\delta^{\mathrm{AB}}=0.060$ & $\delta^{\mathrm{AB}}=0.021$ & \\
\hline$\delta^{\mathrm{AB}}=0.186$ & 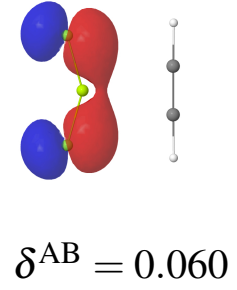 & $\delta^{\mathrm{AB}}=0.066$ & $\begin{array}{c}(0) \\
\delta^{\mathrm{AB}}=0.021\end{array}$ & $\begin{array}{c}\delta^{\mathrm{AB}}=0.028 \\
\end{array}$ \\
\hline$\delta^{\mathrm{AB}}=0.199$ & $\delta^{\mathrm{AB}}=0.057$ & $\delta^{\mathrm{AB}}=0.036$ & $\begin{array}{c}\delta^{\mathrm{AB}}=0.040\end{array}$ & \\
\hline$\delta^{\mathrm{AB}}=0.170$ & $\delta^{\mathrm{AB}}=0.057$ & $\delta^{\mathrm{AB}}=0.029$ & $\delta^{\mathrm{AB}}=0.032$ & $\delta^{\mathrm{AB}}=0.033$ \\
\hline
\end{tabular}

Figure 3: The most highly occupied NAdOs of the $\mathrm{BeF}_{2} \cdots \mathrm{LB}$ systems, with $\mathrm{LB}=\mathrm{C}_{2} \mathrm{H}_{4}{ }^{a}$ ( $1^{\text {st }}$ row), $\mathrm{C}_{2} \mathrm{H}_{2}{ }^{a}$ ( $2^{\text {nd }}$ row), $\mathrm{C}_{2} \mathrm{H}_{4}{ }^{b}$ ( $3^{\text {rd }}$ row), and $\mathrm{C}_{2} \mathrm{H}_{2}{ }^{b}$ (4 ${ }^{\text {th }}$ row). The isosurface shown corresponds to $|\phi|=0.05$ a.u. Their contribution to the $\delta^{\mathrm{AB}}$ delocalization index are shown below. 
together with covalent contributions account for the bonding properties displayed by these interesting systems. We stress that the use of NBOs may skip important delocalization channels that are essential to understand the observed electron fluxes.

\section{One-electron Beryllium bonds}

Beryllium compounds may also become linked via one-electron bonds, which have been rediscovered and analyzed in recent times. We have computed the BeLi and BeNa diatomics, as well as the BeLiBe triatomic system and the $1,8-\mathrm{BeH}$-disubstituted naphtalene anion that was recently proposed as an example of intramolecular Be-Be one-electron bond, ${ }^{55}$ all in their lowest doublet electronic states. Table 5 gathers several IQA properties.

Table 5: Some IQA data for the one-electron bonded LiBe, NaBe, and BeLiBe ${ }^{a}$ linear molecules and the $\mathbf{C}_{\mathbf{1 0}} \mathbf{H}_{\mathbf{6}} \mathbf{B e}_{\mathbf{2}} \mathbf{H}_{\mathbf{2}}^{-}$anion $^{b} . T^{A}, T^{B}, E_{\text {self }}^{A}$, and $E_{\text {self }}^{B}$ in atomic units; $E_{\text {int }}^{A B}, E_{\mathbf{x c}}^{A B}$, and $E_{\mathbf{c l}}^{A B}$ in kcal/mol; $Q$ 's in electrons and $\delta_{\mathrm{AB}}$ in electron pairs.

\begin{tabular}{|lrrrr|rrr|rr|r|}
\hline & $T^{\mathrm{A}}$ & $T^{\mathrm{B}}$ & $E_{\text {self }}^{\mathrm{A}}$ & $E_{\text {self }}^{\mathrm{B}}$ & $E_{\text {int }}^{\mathrm{AB}}$ & $E_{\mathrm{xc}}^{\mathrm{AB}}$ & $E_{\mathrm{cl}}^{\mathrm{AB}}$ & $Q_{\mathrm{A}}$ & $Q_{\mathrm{B}}$ & $\delta^{A \mathrm{~B}}$ \\
\hline $\mathrm{BeLi}$ & 14.723 & 7.418 & -14.557 & -7.285 & -133.36 & -37.47 & -95.89 & -0.707 & 0.708 & 0.466 \\
$\mathrm{BeNa}$ & 14.642 & 161.861 & -14.594 & -161.796 & -54.92 & -35.40 & -19.52 & -0.330 & 0.330 & 0.550 \\
$\mathrm{LiBe}^{a}$ & 7.401 & 14.653 & -7.286 & -14.584 & -68.74 & -19.27 & -49.46 & 0.738 & -0.369 & 0.223 \\
$\mathrm{Be} \cdots \mathrm{Be}^{a}$ & 14.653 & 14.653 & -14.584 & -14.584 & 0.42 & -13.74 & 14.17 & -0.369 & -0.369 & 0.323 \\
$\mathrm{Be} \cdots \mathrm{Be}^{b}$ & 14.316 & 14.316 & -14.114 & -14.114 & 138.43 & -38.75 & 177.18 & 1.258 & 1.258 & 0.293 \\
\hline
\end{tabular}

We first point out that, in agreement with electronegativity arguments, the Be atom gets a negative net charge that accommodates the positively charged alkali atom. This is immediately translated into the Be self-energy, which is progressively destabilized as its negative charge increases. This implies that negatively charging Be has an energy cost, as expected. This conclusion is contrary to that obtained if atomic energies as measured by minus the atomic kinetic energies are used. We warn against using the latter to get chemical insights. Interaction energies are revealing. The total $\mathrm{LiBe}$ interaction is not negligible, about $-130 \mathrm{kcal} / \mathrm{mol}$, and is dominated by a rather large electrostatic component ( $-95 \mathrm{kcal} / \mathrm{mol})$, although covalency cannot be ignored. On moving to $\mathrm{BeNa}$, the covalent component stays almost exactly the same within a couple of $\mathrm{kcal} / \mathrm{mol}$, 
although the electrostatic term is greatly decreased. This is telling us about a rather common electron sharing mechanism, also revealed by the almost $1 / 2$ delocalization index which points toward a single one-electron delocalization channel.

Fig. 4, where $\nabla^{2} \rho(\boldsymbol{r})$ is shown for the BeLi molecule, reveals an easy to recognize pattern in highly ionic molecules. The Li atom has lost its valence shell, and the $\nabla^{2} \rho<0$ region of the Be atom is now heavily polarized toward the Li moiety. We have superimposed the electrostatic potential mapped onto the $\rho=0.03$ isosurface to show how the standard concepts used in intermolecular interactions can be directly translated to the present examples, although we use a much larger value of $\rho$ in this case. The Be atom has a core-like positive ESP region that corresponds well to a laplacian depletion zone, with electron rich regions to its sides. This agrees with a $s-p_{z^{-}}$ like hybridization of the one-electron bond that will come out clear in the following. Notice that the polarization of the $\operatorname{Be} \nabla^{2} \rho<0$ domain coincides with that of the ESP. On the contrary the Li core corresponds to a slightly polarized positive ESP region which faces the electron rich Be. This picture qualitatively points toward an important role of electrostatics in BeLi bonding. IQA easily quantifies it. Similar images are found for the BeNa case.

Even more interesting is the consideration of the BeLiBe triatomic. In this case, the sum of both $E_{\mathrm{xc}}^{\mathrm{LiBe}}$ energies adds to almost exactly the same value as in the $\mathrm{LiBe}$ diatomic. The same can be said about the total interaction energy. Each of the two LiBe interactions is halved with respect to $\mathrm{LiBe}$. In chemical terms, we are sharing the electron between three regions, as we will see, and the delocalization index between each LiBe pair is again halved. This consistency is one of the important properties of IQA. In BeLiBe, the one-electron bond is tricentric: the three-center delocalization index $\left(\delta^{\mathrm{BeLiBe}}=0.186\right)^{41}$ is one of the largest ever reported.

More insight about the electronic structure of these compounds is obtained by examining their EDFs. Table 6 summarizes de probability of the different real space resonance structures. In BeLi and $\mathrm{BeNa}$ it is rather clear that, except residual contributions, the distribution is dominated by two structures. This is a obviuos sign of a one-electron delocalization. In BeLi, the delocalized electron is heavily polarized toward the Be atom, $p\left(\mathrm{n}_{\mathrm{Be}}=5, \mathrm{n}_{\mathrm{Li}}=2\right)=0.7$. This polarization 


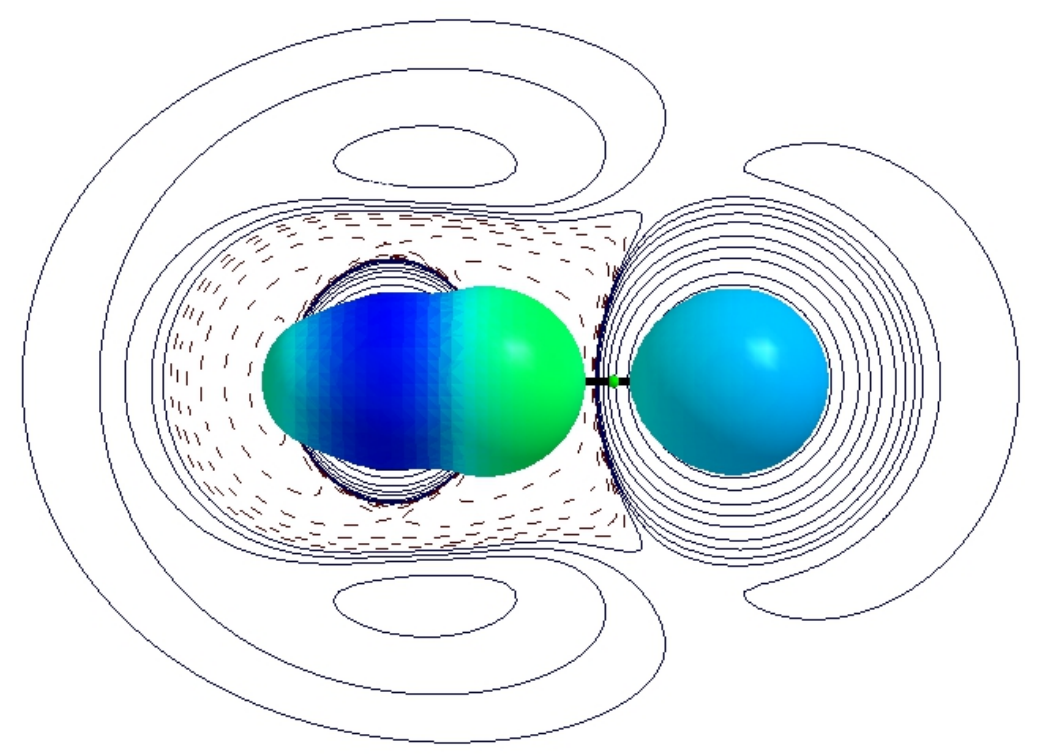

Figure 4: $\nabla^{2} \rho(\boldsymbol{r})$ for the BeLi molecule in a plane containing the nuclei. An isosurface of $\rho=0.03$ a.u. with the electrostatic potential mapped onto it has been superimposed. The Be atom is on the left side of the figure, and the BeLi bond critical point of the electron density is marked as a small green sphere.

Table 6: Electron distribution functions for the one-electron bonded LiBe, NaBe, and BeLiBe linear molecules. The atoms are labelled in the order in which they are written. In this sense, $n_{A}, n_{B}$ refer to the populations in Be and $\mathrm{Li}$ in the BeLi moiety. All data in atomic units.

\begin{tabular}{|rrr|rrr|rrrr|}
\hline \multicolumn{3}{|c|}{$\mathrm{BeLi}$} & \multicolumn{4}{|c|}{$\mathrm{BeNa}$} & \multicolumn{4}{c|}{ BeLiBe } \\
\hline$n_{A}$ & $n_{B}$ & $p\left(n_{A}, n_{B}\right)$ & $n_{A}$ & $n_{B}$ & $p\left(n_{A}, n_{B}\right)$ & $n_{A}$ & $n_{B}$ & $n_{C}$ & $p\left(n_{A}, n_{B}, n_{C}\right)$ \\
\hline 5 & 2 & 0.710 & 4 & 11 & 0.619 & 4 & 2 & 5 & 0.370 \\
4 & 3 & 0.275 & 5 & 10 & 0.354 & 5 & 2 & 4 & 0.370 \\
3 & 4 & 0.009 & 3 & 12 & 0.025 & 4 & 3 & 4 & 0.219 \\
6 & 1 & 0.004 & 6 & 9 & 0.001 & 5 & 3 & 3 & 0.013 \\
& & & & & & 3 & 3 & 5 & 0.013 \\
\hline
\end{tabular}


decreases in BeNa. The BeLiBe data show again that the bonding electron is delocalized among the three atoms, slightly more polarized toward the Be ends and providing a very neat image of a three-center one-electron bond.
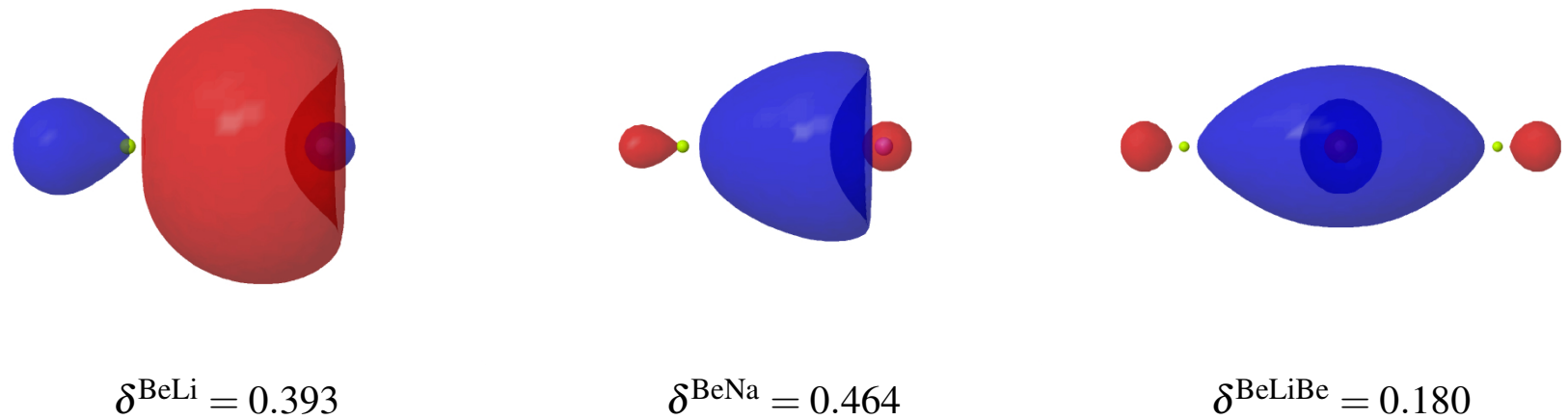

$$
\delta^{\mathrm{BeNa}}=0.464
$$

$$
\delta^{\mathrm{BeLiBe}}=0.180
$$

Figure 5: One-electron NAdOs for the BeLi (left), BeNa (middle), and BeLiBe (right) systems. The isosurface shown corresponds to $|\phi|=0.05$ a.u. Their contribution to the two-center (BeLi and $\mathrm{BeNa}$ ) or three-center delocalization indices (BeLiBe) are shown below.

A pictorial glimple of the one-electron bonds is shown in Fig. 5 where the main NAdO component is shown. Only one exchange channel (NAdO) accounts for almost all of the final electron delocalization. The only exception is BeLi, where a second smaller contribution, basically a polarized Be $2 s$ orbital is also found. The one-electron bond is a combination of Be $s p_{z}$-like hybrids and polarized Li $2 s$ or Na $3 s$ functions, as expected.

We have also computed the 1,8- $\mathrm{BeH}$ disubstituted naphtalene anion, in which a relatively strong one-electron intramolecular Be-Be bond was recently reported. ${ }^{55}$ In agreement with previous knowledge, a bond critical point between the Be atoms appears, with $\rho=0.033$ a.u. and $\nabla^{2} \rho=-0.041$ a.u. As seen in Table 5 both Beryllium atoms are considerably positively charged, this leading to a large electrostatic destabilization. We thus have in this case a homonuclear-like interaction, which is only partially stabilized by a covalent term of about $-39 \mathrm{kcal} / \mathrm{mol}$, with a delocalization index close to that shown in BeLiBe. To understand the nature of the $\mathrm{Be} \cdots \mathrm{Be}$ stabilization it is necessary to consider the interaction of the two $-\mathrm{C}_{\text {naph }} \mathrm{BeH}$ fragments, where negatively charged groups (like the $\mathrm{H}$ atoms) compensate the Be...Be electrostatic destabilization. This can be done easily in IQA, resulting in a total interaction energy between the fragments of 
about $-62 \mathrm{kcal} / \mathrm{mol}$, with only $-8 \mathrm{kcal} / \mathrm{mol}$ coming from electrostatic interaction. Actually, the two $-\mathrm{C}_{\text {naph }} \mathrm{BeH}$ moieties display, as a whole, a net charge of only -0.469 extra electrons, meaning that half the anionic charge is delocalized between the two $-\mathrm{C}_{\text {naph }} \mathrm{BeH}$ fragments. All this tells about a rather important energy stabilization coming from the formation of the Be-Be bond.

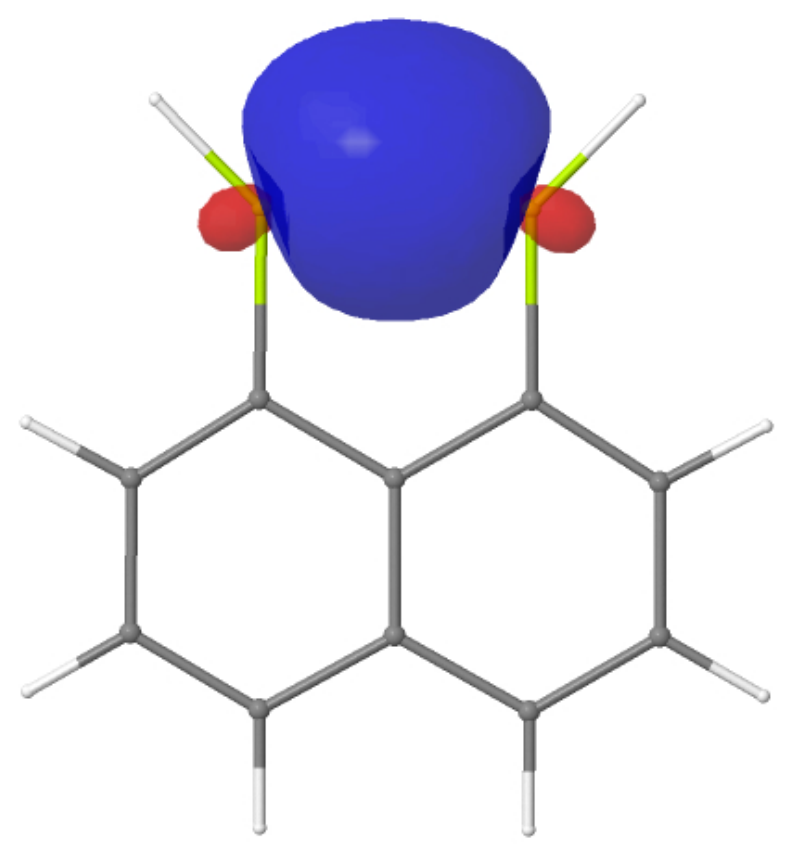

Figure 6: One-electron Be-Be NAdO for the $\mathrm{C}_{10} \mathrm{H}_{6} \mathrm{Be}_{2} \mathrm{H}_{2}^{-}$anion. The isosurface shown corresponds to $|\phi|=0.05$ a.u.

Fig. 6 shows the Be-Be one-electron NAdO obtained from our DFT calculation. It shows a very clear in-phase combination of $s p^{2}$-like hybrids, in agreement with previous knowledge. It accounts for most of the Be-Be delocalization index.

Altogether, these examples show that the same tools that can be used in the case of intermolecular or weaker interactions apply equally well to more strongly bound systems. Taking BeLi, for instance, charge transfer is not in the model, but a very real phenomenon. The probability that taking a snapshot of the system we find a $\mathrm{Be}^{-}$moiety is 0.7 . At the same time, the ESP, the density, or the laplacian of the density, all point in this direction. Evaluation of the exact electrostatics coming from the in-the-molecule electron density provides an important part of the BeLi interac- 
tion energy, but falls short of its total value. The lacking ingredient is delocalization or covalency, which corrects the electrostatic interaction because some electrons that contribute to the density (and to $E_{\mathrm{cl}}$ ) are counted several times due to their wandering nature, in other words, because the pair density is not contained in the density. Analyzing in detail how delocalization takes place we can go back to standard molecular orbital arguments, all from observable quantities. If this is accepted, smaller intermolecular charge transfers do also have to, without models.

\section{Conclusions}

We have used in this contribution several recent quantum chemical topology tools, including the interacting quantum atoms (IQA) approach and the effective one-electron pictures provided by two-fragment natural adaptive orbitals or electron distribution functions, to shed new light on the nature of the interactions in some typical Beryllium-bonded systems. These tools add to the traditional local topological approaches, complementing them. The real space energetic partitioning of IQA shows that, in agreement with previous works, it is the electrostatic interaction between the bonded fragments that accounts for the basis energetics as well as conformational preferences in the compounds examined. However, in the absence of delocalization (covalent) contributions, the deformation energy of the fragments is not overcome by electrostatics. In this sense, and similarly to what was found in simple hydrogen-bonded systems, ${ }^{53}$ covalency is essential for the stability of the aggregates. An overlooked aspect related to the total charge transfer has been analyzed in detail. This real space charge transfer is free from the criticisms posed by Politzer et al. ${ }^{10}$. Our results clearly show that there exist important delocalization channels that are not properly accounted for by the natural bond orbital (NBO) formalism, involving $\sigma$ back-donation from $\mathrm{BeX}_{2}$ moieties to the Lewis base which can even be dominant, leading to negative net LB charges. The strength of the different forward and back-bonding channels is predictable from the characteristics of the fragments involved, leading to a chemically appealing rationalization of conformational preference. An analysis of some toy Beryllium containing molecules displaying one-electron bonds has 
also been undertaken. EDFs and NAdOs show that the one-electron bonding model accurately describes their bonding.

\section{Supporting Information}

Optimized geometries, Electron Distribution Functions (EDFs), and Natural Adaptive Orbitals (NAdOs) of the molecules studied in this work.

\section{Acknowledgments}

We thank the Spanish MINECO, grant CTQ2015-65790-P, the FICyT, grant GRUPIN14-049, and the European Union FEDER for funding. F. J.-G. gratefully acknowledge financial support from the Spanish MINECO, grant BES-2016-076986.

\section{References}

(1) Scnheider, H.-J. Binding Mechanisms in Supramolecular Complexes. Angew. Chem. Int. Ed. 2009, 48, 3924-3977.

(2) Hobza, P.; Müller-Dethlefs, K. Non-Covalent Interactions: Theory and Experiment; Royal Society of Chemistry: Cambridge, 2010.

(3) Novoa, J., Ed. Intermolecular Interactions in Crystals: Fundamentals of Crystal Engineering; The Royal Society of Chemistry: Cambridge, 2017.

(4) Jeffrey, G. An Introduction to Hydrogen Bonding; 1997: New York, Oxford University Press.

(5) Desiraju, G.; Steiner, T. The Weak Hydrogen Bond in Structural Chemisry and Biology; Oxford University Press: New York, 1999.

(6) Buckingham, A. D.; Fowler, P. W.; Jutson, J. M. Theoretical Studies of van der Waals Molecules and Intermolecular Forces. Chem. Rev. 1988, 88, 963-988. 
(7) Spackman, M. A. A Simple Quantitative Model of Hydrogen Bonding. J. Chem. Phys. 1986, $85,6587-6601$.

(8) Weinhold, F. Nature of H-Bonding in Clusters, Liquids, and Enzymes: an Ab Initio, Natural Bond Orbital Perspective. J. Mol. Struct. 1997, 398-399, 181-197.

(9) Isaacs, E. F.; Shukla, A.; Platzman, P. M.; Hamann, D. R.; Barbiellini, B.; Tulk, C. A. Covalency of the Hydrogen Bond in Ice: A Direct X-Ray Measurement. Phys. Rev. Lett. 1999, 82, $600-603$.

(10) Politzer, P.; Murray, J. S.; Clark, T. Mathematical Modeling and Physical Reality in Noncovalent Interactions. J. Mol. Model. 2015, 21, 52.

(11) Murray, J. S.; Politzer, P. The Electrostatic Potential: An Overview. WIREs Comput. Mol. Sci. 2011, 1, 153-163.

(12) Weinhold, F.; Landis, C. Valency and Bonding. A Natural Bond Orbital Donor-Acceptor Perspective; Cambridge Univ. Press, 2005.

(13) Arunan, E.; Desiraju, G. R.; Klein, R. A.; Sadlej, J.; Scheiner, S.; I., A.; C., C. D.; Crabtree, R. H.; Dannenberg, J. J.; Hobza, P. et al. Defining the Hydrogen Bond: An Account. Pure Appl. Chem. 2011, 1619-1636.

(14) Yañez, M.; Sanz, P.; Mó, O.; Alkorta, I.; Elguero, J. Beryllium Bonds, Do They Exist? J. Chem. Theory Comput. 2009, 5, 2763-2771.

(15) Fernández Villanueva, E.; Mó, O.; Yañez, M. On the Existence and Characterization of $\pi$-Beryllium Bonds. Phys. Chem. Chem. Phys. 2014, 16, 17531-17536.

(16) Brea, O.; Mó, O.; Yañez, M.; Alkorta, I.; Elguero, J. Creating $\sigma$-Holes through the Formation of Beryllium Bonds. Chemistry - A European Journal 2015, 21, 12676-12682.

(17) Gimarc, B. M. Molecular Structure and Bonding. The Qualitative Molecular Orbital Approach; Academic Press: New York, 1979. 
(18) Su, P.; Li, H. Energy Decomposition Analysis of Covalent Bonds and Intermolecular Interactions. J. Chem. Phys. 2009, 131, 014102.

(19) Bader, R. F. W. Atoms in Molecules; Oxford University Press: Oxford, 1990.

(20) D., B. A.; Edgecombe, K. E. A Simple Measure of Electron Localization in Atomic and Molecular Systems. J. Chem. Phys. 1990, 92, 5397-5403.

(21) Mitoraj, M.; Michalak, A. Natural Orbitals for Chemical Valence as Descriptors of Chemical Bonding in Transition Metal Complexes. J. Mol. Model. 2007, 13, 347-355.

(22) Michalak, A.; Mitoraj, M.; Ziegler, T. Bond Orbitals from Chemical Valence Theory. J. Phys. Chem A. 2008, 112, 1933-1939.

(23) Menéndez, M.; Álvarez-Boto, R.; Francisco, E.; Martín Pendás, A. One-Electron Images in Real Space: Natural Adaptive Orbitals. J. Comput. Chem. 2015, 36, 833-843.

(24) Chen, X.; Grammer, J.; Lawson, J.; Cooke, R.; Pate, R., E. Yount A Novel Restricted Photoaffinity Spin-Labeled Non-Nucleoside ATP Analogue as a Covalently Attached Reporter Group of the Active Site of Myosin Subfragment 1. Biochemistry 2002, 41, 2609-2620.

(25) Shibuya, H.; Kondo, K.; Kimura, N.; Maruta, S. Formation and Characterization of Kinesin.ADP.Fluorometal Complexes. J. Biochem. 2002, 132, 573-579.

(26) Danko, S.; Yamasaki, K.; Daiho, T.; Suzuki, H. Distinct Natures of Beryllium Fluoridebound, Aluminum Fluoride-bound, and Magnesium Fluoride-bound Stable Analogues of an ADP-insensitive Phosphoenzyme Intermediate of Sarcoplasmic Reticulum $\mathrm{Ca}^{2+}$-ATPase CHANGES IN CATALYTIC AND TRANSPORT SITES DURING PHOSPHOENZYME HYDROLYSIS. J. Biol. Chem. 2004, 279, 14991-14998.

(27) Sauna, Z.; Nandigama, K.; Ambudkar, S. Exploiting Reaction Intermediates of the ATPase Reaction to Elucidate the Mechanism of Transport by P-glycoprotein (ABCB1). J. Biol. Chem. 2006, 281, 26501-26511. 
(28) Tiago, T.; Simao, S.; Aureliano, M.; Martin-Romero, F.; Gutierrez-Merino, C. Inhibition of Skeletal Muscle S1-Myosin ATPase by Peroxynitrite. Biochemistry 2006, 45, 3794-3804.

(29) Orban, J.; Lorinczy, D.; Hild, G.; Nyitrai, M. Noncooperative Stabilization Effect of Phalloidin on $\mathrm{ADP}_{\mathrm{BeF}}$ - and $\mathrm{ADP}^{\mathrm{AlF}} 4$ - Actin Filaments. Biochemistry 2008, 47, 4530-4534.

(30) Liu, F.; Putnam, A.; Jankowsky, E. ATP hydrolysis is required for DEAD-box protein recycling but not for duplex unwinding. Proc. Natl. Acad. Sci. 2008, 105, 20209-20214.

(31) Barbaro, P.; Cecconi, F.; Dakternieks, D.; Dominguez, S.; Duthie, A.; Ghilardi, C.; Midollini, S.; Orlandini, A.; Vacca, A. Beryllium(II) Complexes of the Kläui Tripodal Ligand Cyclopentadienyltris(diethylphosphito-P)cobaltate(-). Inorg. Chem. 2003, 40, 2725-2729.

(32) Conejo, M.; Fernández, R.; Carmona, E.; Andersen, R.; Gutiérrez-Puebla, E.; Monge, M. Synthetic, Reactivity, and Structural Studies on Half-Sandwich $\left(\eta^{5}-\mathrm{C}_{5} \mathrm{Me}_{5}\right) \mathrm{Be}$ and Related Compounds: Halide, Alkyl, and Iminoacyl Derivatives. Chem. Eur. J. 2003, 9, 4462-4471.

(33) Schmidbaur, H. Coord. Chem. Rev. 2001, 215, 223-241.

(34) Scott, B.; McCleskey, T.; Chaudhary, A.; Hong-Geller, E.; Gnanakaran, S. Chem. Commun. 2008, 371, 2837-2847.

(35) Gatti, C., Macchi, P., Eds. Modern Charge-Density Analysis; Springer, Dordrecht., 2012.

(36) Matta, C. F., Boyd, R. J., Eds. The Quantum Theory of Atoms in Molecules; Wiley-VCH Verlag GmbH \& Co. KGaA, 2007.

(37) Francisco, E.; Martín Pendás, A.; García-Revilla, M.; Álvarez Boto, R. A Hierarchy of Chemical Bonding Indices in Real Space from Reduced Density Matrices and Cumulants. Comput. Theor. Chem. 2013, 1003, 71-78.

(38) Blanco, M. A.; Martín Pendás, A.; Francisco, E. Interacting Quantum Atoms: A Correlated Energy Decomposition Scheme based on the Quantum Theory of Atoms in Molecules. J. Chem. Theory Comput. 2005, 1, 1096-1109. 
(39) Francisco, E.; Martín Pendás, A.; Blanco, M. A. A Molecular Energy Decomposition Scheme for Atoms in Molecules. J. Chem. Theory Comput. 2006, 2, 90-102.

(40) Cancès, E.; Keriven, R.; Lodier, F.; Savin, A. How Electrons Guard the Space: Shape Optimization with Probability Distribution Criteria. Theor. Chem. Acc. 2004, 111, 373-380.

(41) Francisco, E.; Martín Pendás, A.; Blanco, M. A. Electron Number Probability Distributions for Correlated Wave Functions. J. Chem. Phys. 2007, 126, 094102.

(42) Martín Pendás, A.; Francisco, E.; Blanco, M. A. Spin Resolved Electron Number Distribution Functions: How spins Couple in Real Space. J. Chem. Phys. 2007, 127, 144103.

(43) Jayatilaka, D.; Grimwood, D. J. Wavefunction Derived from Experiment. I. Motivation and Theory. Acta Cryst. A. 2001, 57, 76-86.

(44) Grabowsky, S.; Genoni, A.; Bürgi, H.-B. Quantum Crystallography. Chem. Sci. 2017, 8, 4159-4176.

(45) Schmidt, M. W.; Baldridge, K. K.; Boatz, J. A.; Elbert, S. T.; Gordon, M. S.; Jensen, J. H.; Koseki, S.; Matsunaga, N.; Nguyen, K. A.; Su, S. et al. General Atomic and Molecular Electronic Structure System. Journal of Computational Chemistry 1993, 14, 1347-1363.

(46) Sun, Q.; Berkelbach, T. C.; Blunt, N. S.; Booth, G. H.; Guo, S.; Li, Z.; Liu, J.; McClain, J.; Sharma, S.; Wouters, S. et al. The Python-based Simulations of Chemistry Framework (PySCF). 2017,

(47) Francisco, E.; Casals-Sainz, J. L.; Rocha-Rinza, T.; Martín Pendás, Partitioning the DFT Exhange-Correlation Energy in Line with the Interacting Quantum Atoms Approach. Theor. Chem. Acc. 2016, 135, 170-177.

(48) Knizia, G.; Chan, G. K.-L. Density Matrix Embedding: A Simple Alternative to Dynamical Mean-Field Theory. Phys. Rev. Lett. 2012, 109, 186404. 
(49) Martín Pendás, A.; Francisco, E. PROMOLDEN: A QTAIM/IQA code (Available from the authors upon request by writing to ampendas@uniovi.es).

(50) Francisco, E.; Martín Pendás, A. Electron Number Distribution Functions from Molecular Wavefunctions. Version 2. Comput. Phys. Commun. 2014, 185, 2663-2682.

(51) Francisco, E.; Martín Pendás, A. DENMAT: Density Matrices and Natural Adaptive Orbitals (Available from the authors upon request by writing to evelio@uniovi.es).

(52) Eskandari, K. Nature of Beryllium Bonds in View of Interacting Quantum Atoms and Natural Energy Decomposition Analyses. Comput. Theor. Chem. 2016, 1090, 74-79.

(53) Martín Pendás, A.; Blanco, M. A.; Francisco, E. The Nature of the Hydrogen Bond: A Synthesis from the Interacting Quantum Atoms Picture. J. Chem. Phys. 2006, 125, 184112.

(54) Martín Pendás, A.; Blanco, M. A.; Francisco, E. Steric Repulsions, Rotation Barriers, and Stereoelectronic Effects: A Real Space Perspective. J. Comput. Chem. 2009, 30, 98-109.

(55) Brea, O.; Mó, O.; Yañez, M.; Alkorta, I.; Elguero, J. On the Existence of Intramolecular One-Electron Be-Be Bonds. Chem. Commun. 2016, 52, 9656-9659. 